\title{
Retropharyngeal Hematoma Secondary to Vomiting in a Healthy Adult: A Case Report
}

\author{
Hyokrae Cho
}

\begin{abstract}
Nontraumatic spontaneous retropharyngeal hematoma is rarely encountered. Clinical diagnosis of retropharyngeal hematoma can be difficult and may be infrequently misdiagnosed with acute pharyngitis. A careful history taking is also an important process to diagnose this disease entity. Because of its rarity and difficulty of early diagnosis, retropharyngeal hematoma may rapidly progress to airway compromise. A 51-year-old woman suddenly developed neck pain and dyspnea after vomiting. Examination showed anterior bulging of the posterior oropharyngeal wall without ecchymosis around neck. No underlying medical conditions, medications, or trauma were found. Cervical magnetic resonance imaging revealed retropharyngeal hematoma collection from nasopharynx to laryngopharynx. We report a case of spontaneous retropharyngeal hematoma with increased intrathoracic pressure caused by vomiting.
\end{abstract}

Keywords: Retropharyngeal hematoma; Airway; Intrathoracic pressure

\section{Introduction}

Retropharyngeal hematoma occurs infrequently, whereas spontaneous case is also rarer among them. There are several factors involved in its etiology. By comparison, that disease needs emergency attention and critical airway care in the setting of airway obstruction, leading to more fatal outcome.

The diagnosis can be difficult and a lot of clinicians often overlook this disease entity at their outpatient clinic. The early recognition of this pathology is very important with use of magnetic resonance imaging (MRI). Signal changes caused by blood products which are visible on the MR images suggest the diagnosis of retropharyngeal hematoma. With a review of the literature, the author presents a rare case of a 51-year-old

Manuscript accepted for publication January 05, 2015

Department of Neurosurgery, Kosin University Gospel Hospital, Pusan, Korea.Email: drchr@hanmail.net

doi: http://dx.doi.org/10.14740/jmc2038w woman with spontaneous retropharyngeal hematoma after vomiting.

\section{Case Report}

A 51-year-old woman was admitted to our hospital in September 2014 due to 3 days history of dull neck pain and orthopnea without history of preexisting neck disease. Especially, she felt dyspnea whenever she flexed her neck. Vomiting was suddenly developed due to indigestion just before she visited our hospital. Previous medical history was non-specific. On general examination, this patient looked healthy. The neck was nontender and there was no significant limitation of neck movement. Her vital sign was also stable.

A fiberoptic laryngoscopic examination revealed a slight anterior bulging of the posterior pharyngeal wall with preserved glottis space (Fig. 1). Hematology evaluation showed a hemoglobin of $13.6 \mathrm{~g} / \mathrm{dL}$, white blood cell count (per $\mathrm{mm}^{3}$ ) of 6,800 , platelet count of $278,000 / \mathrm{dL}$, erythrocyte sedimentation rate (ESR) of $15 \mathrm{~mm} / \mathrm{h}$ and C-reactive protein of $1 \mathrm{mg} / \mathrm{L}$. Biochemical analysis, prothrombin time (PT), partial thromboplastin time (PTT) and bleeding time were normal. Lateral soft tissue radiography of the neck showed increased thickness of prevertebral soft tissue: $11.6 \mathrm{~mm}$ at $\mathrm{C} 1,8.1 \mathrm{~mm}$ at $\mathrm{C} 2,9.8$ $\mathrm{mm}$ at $\mathrm{C} 3$ and $9.8 \mathrm{~mm}$ at $\mathrm{C} 4$ (Fig. 2). The airway was not significantly narrowed.

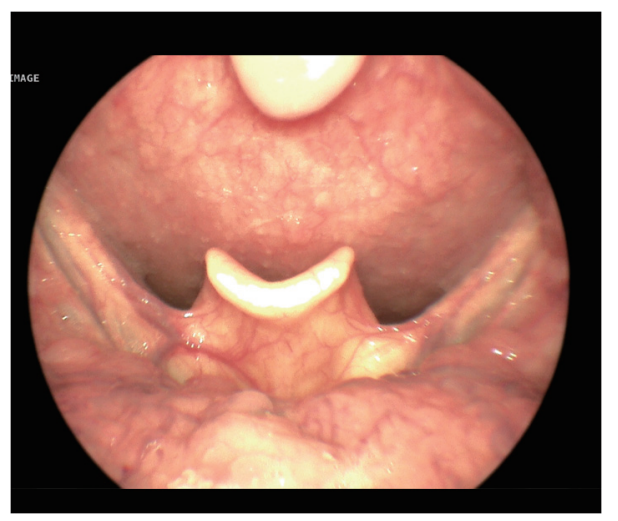

Figure 1. The laryngoscopic finding. The feature shows anterior bulging of posterior oropharynx wall. 


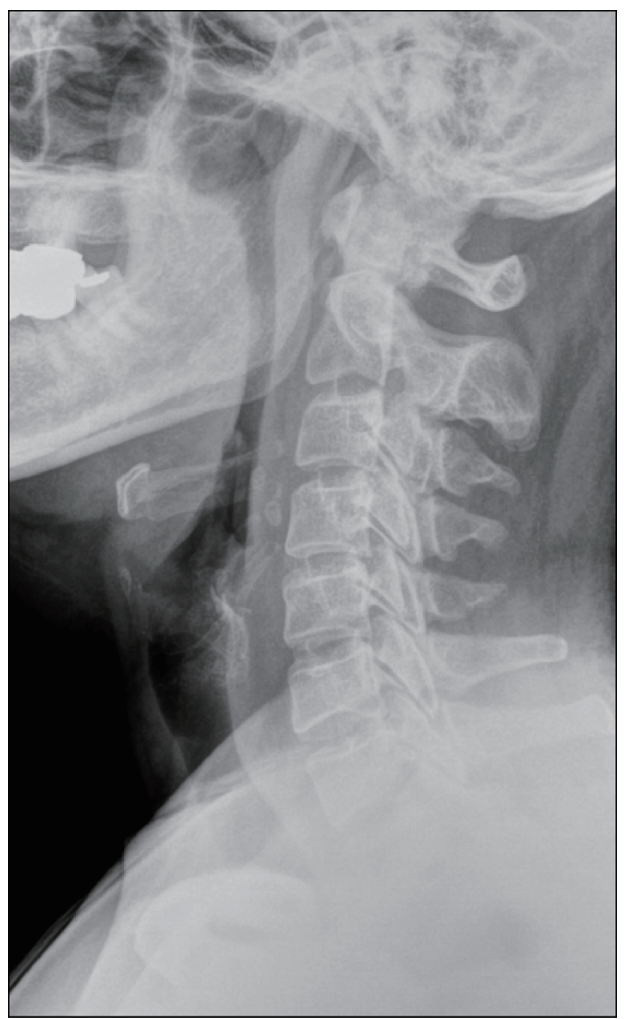

Figure 2. Lateral neck X-ray shows a widening of the prevertebral space.

And MRI was performed (Fig. 3). The image sequences disclosed a retropharyngeal collection that exhibited high signal on routine T2-weighted image and fluid-attenuated inversion recovery (FLAIR) image. Low signal was also found at T1-weighted image. Above mentioned image sequences disclosed typical signal characteristics of an acute hematoma. The patient was managed conservatively with steroids and there was complete resolution of neck pain and orthopnea. On the third day, her symptom almost all resolved. The patient was discharged within 7 days.

\section{Discussion}

Anatomically, the retropharyngeal space is located between the middle and deep layers of the deep cervical fascia and presents ventral, dorsal and lateral borders. The ventral border of the retropharyngeal space is constituted by the buccopharyngeal fascia and the superior pharyngeal constrictor, the dorsal border by the alar and prevertebral fascia and lateral border by the carotid sheaths and other deep fascial spaces, respectively. That is largest interfascial compartment. Bleeding in these potential spaces can be an immediate life-threatening emergency with potential for airway compromise due to the wide extension of this potential space from the skull base to the level of carina [1]. As a result, all physicians involved in the management of airway emergencies should be aware of this rare condition. In our case, retropharyngeal swelling and tracheal compression were not found. Fortunately, respiratory distress was not progressed.

The frequency of spontaneous retropharyngeal hematoma is clearly unknown. Spontaneous retropharyngeal hematoma is defined by the absence of any clear etiology. There are only a few case reports in the recent literature [2]. Retropharyngeal hematomas are, although extremely uncommon, associated with a range of conditions including cervical trauma, anticoagulation, bleeding diathesis, following internal jugular vein cannulation, arteriography and carotid sinus massage $[3,4]$.

Precipitating factors, such as episodes of coughing, sneezing, straining, and vomiting, indirectly contribute to a retropharyngeal hematoma. A proposed mechanism is that increased intrathoracic pressure causes increased jugular pressure transmitted to tributary valveless veins leading to rupture of bridging veins [1]. In traumatic cases, the hematoma is thought to be caused by a tear in the anterior longitudinal ligament, but it can also be due to bleeding from a cervical spinal fracture. However, our patient presented with acute onset of mild neck pain and dyspnea after vomiting. No apparent trau-
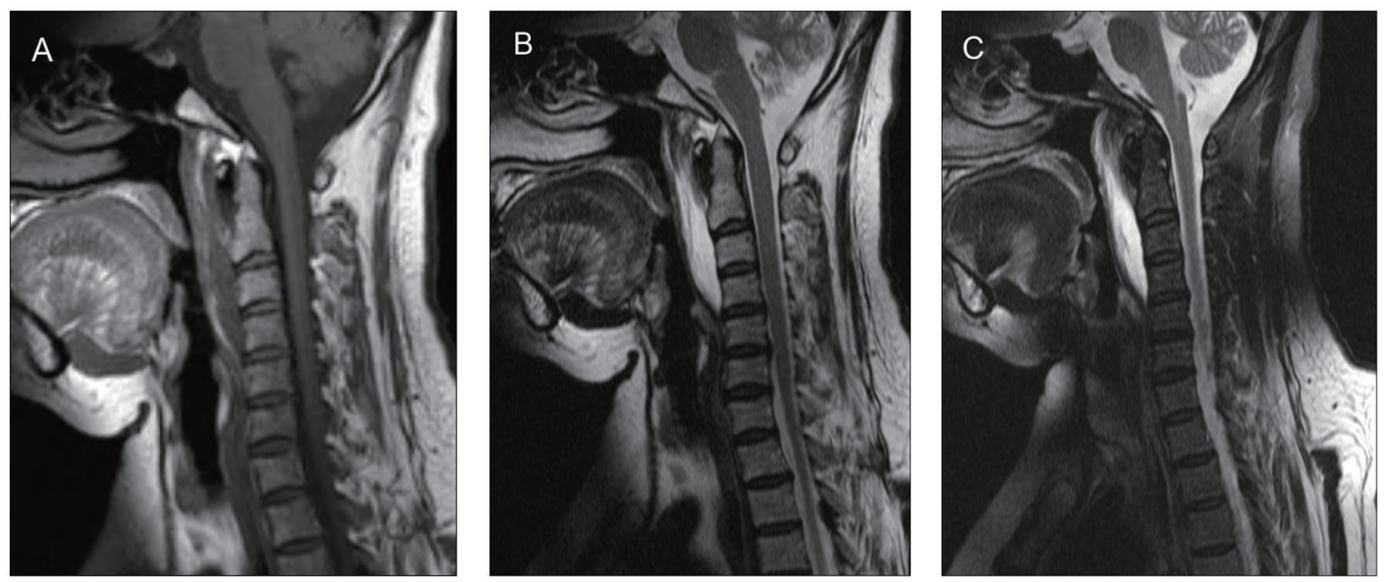

Figure 3. Midsagittal T1-weighted image (A), T2-weighted image (B) and FLAIR image (C). The T1-, T2-weighted image and FLAIR image are consistent with the predominant deoxyhemoglobin. 
ma history was noted. Vomiting was only predisposing factor for triggering hemorrhage.

The diagnosis of retropharyngeal hematoma can be difficult because symptom and sign are various and non-specific. Clinically, it may manifest as sore throat, neck pain, dysphagia, dyspnea and a hoarse voice. In case of trauma, the Capps triad has been described: tracheal and esophageal compression, ventral displacement of the trachea, and subcutaneous ecchymosis over the neck and anterior chest wall [5].

The diagnosis is aided by X-ray, computed tomography, or MRI. The thickness of the prevertebral soft tissue is helpful in detecting a retropharyngeal hematoma. The thickening of prevertebral soft tissue should be correlated with the clinical findings. Lateral X-ray of the neck is a simple and useful tool for measuring the prevertebral soft tissue. Hauq et al reviewed a total of 131 lateral soft tissue radiographs in various age groups, of which 86 radiographs were normal. In this study, the mean thickness in the retropharyngeal region ranged from 6.2 $\pm 3.0 \mathrm{~mm}$ in the infant to $3.7 \pm 1.0 \mathrm{~mm}$ in the adult [6]. Rojas et al reported that the upper limits of normal range for thickness of prevertebral soft tissue in multidetector CT were $8.5 \mathrm{~mm}$, $6 \mathrm{~mm}, 7 \mathrm{~mm}, 18 \mathrm{~mm}$ and $18 \mathrm{~mm}$ at $\mathrm{C} 1, \mathrm{C} 2, \mathrm{C} 3, \mathrm{C} 6$ and $\mathrm{C} 7$, respectively [7]. In our case, thickness of the prevertebral soft tissue was also mildly increased to $11.6 \mathrm{~mm}$ at $\mathrm{C} 1,8.1 \mathrm{~mm}$ at $\mathrm{C} 2,9.8 \mathrm{~mm}$ at $\mathrm{C} 3$ and $9.8 \mathrm{~mm}$ at $\mathrm{C} 4$ compared to these reference values. These findings allowed further diagnostic workup by $\mathrm{CT}$ and/or MRI.

MRI offers multiplanar anatomic display and the tissue characterization in head and neck. The differential diagnosis of retropharyngeal lesions can be possible with use of contrast. Also, the MRI allows more specific diagnosis about blood products in different stages of evolution, because of their paramagnetic signal properties which change over the time depending on their dominant component (acute deoxyhemoglobins, subacute intra- or extracellular methemoglobins and chronic hemichromes) $[5,8]$. Our patient also exhibited hypointensity on T1-sequneces and high intensity on T2-weighted sequences and FLAIR image. The enhancing MRI was not carried out because MR findings of this patient were compatible to acute retropharyngeal hematoma although retropharyngeal infection was also considered as a differential diagnosis. Clinical and laboratory findings such as fever and dysphagia, leukocytosis and elevated ESR were necessary to consider infection.

The prognosis is good if recognized and treated promptly. Despite the absence of respiratory distress at the time of admission, emergent airway management with either rapid intubation or tracheostomy may become necessary. Bleeding into the retropharyngeal space is serious because the anatomic peculiarity of the pharyngeal muscles offers no resistance to the expanding hematoma. Compression of the arytenoids cartilages can occur, closing the vocal and obstructing the airway [4].

However, surgical exploration and transoral drainage should be only considered for large hematoma, especially for those rapidly expanding. Most cases of small or moderate hematomas can be resolved with a conservative management [9]. Fortunately, symptom and sign of this patient were rapidly improved after medication.

\section{Conclusion}

Our case illustrates a presentation of spontaneous retropharyngeal hematoma after vomiting in the form of no airway compromise. Physician should be aware that retropharyngeal hematoma may be early misdiagnosed and could lead to airway obstruction. An early lateral neck X-ray and MRI are essential for diagnosis and management of this rare disease. This knowledge is very important for managing retropharyngeal hematoma properly.

\section{References}

1. Dedouit F, Grill S, Guilbeau-Frugier C, Savall F, Rouge D, Telmon N. Retropharyngeal hematoma secondary to cervical spine surgery: report of one fatal case. J Forensic Sci. 2014;59(5):1427-1431.

2. Gao R, Shao A, Dorman B. Spontaneous retropharyngeal haematoma: report of a case. ANZ J Surg. 2011;81(10):756.

3. Senthuran S, Lim S, Gunning KE. Life-threatening airway obstruction caused by a retropharyngeal haematoma. Anaesthesia. 1999;54(7):674-678.

4. Chin KW, Sercarz JA, Wang MB, Andrews R. Spontaneous cervical hemorrhage with near-complete airway obstruction. Head Neck. 1998;20(4):350-353.

5. Munoz A, Fischbein NJ, de Vergas J, Crespo J, Alvarez-Vincent J. Spontaneous retropharyngeal hematoma: diagnosis by $\mathrm{mr}$ imaging. AJNR Am J Neuroradiol. 2001;22(6):1209-1211.

6. Haug RH, Wible RT, Lieberman J. Measurement standards for the prevertebral region in the lateral soft-tissue radiograph of the neck. J Oral Maxillofac Surg. 1991;49(11):1149-1151.

7. Rojas CA, Vermess D, Bertozzi JC, Whitlow J, Guidi C, Martinez CR. Normal thickness and appearance of the prevertebral soft tissues on multidetector CT. AJNR Am J Neuroradiol. 2009;30(1):136-141.

8. Bakshi R, Kamran S, Kinkel PR, Bates VE, Mechtler LL, Janardhan V, Belani SL, et al. Fluid-attenuated inversionrecovery MR imaging in acute and subacute cerebral intraventricular hemorrhage. AJNR Am J Neuroradiol. 1999;20(4):629-636.

9. Senel AC, Gunduz AK. Retropharyngeal hematoma secondary to minor blunt neck trauma: case report. Rev Bras Anestesiol. 2012;62(5):731-735. 